\title{
Environment Monitoring for Transmission Electron Microscopy
}

\author{
Denis Fellmann, Rachel Banez, Bridget Carragher and Clinton S. Potter
}

National Resource for Automated Molecular Microscopy and Department of Cell Biology, The Scripps Research Institute, La Jolla, CA 92037

The microscopy suite at the Center for Integrative Molecular Biosciences at the Scripps Research Institute includes a set of six rooms that have been specifically designed for Transmission Electron Microscopy of vitreous ice specimens. Design considerations focused on vibration and acoustic isolation, thermal stability and low relative humidity [1]. When acquiring images from specimens preserved in vitreous ice and transferred to the microscope using a side entry holder the thermal stability of the environment is particularly critical. Any fluctuations in temperature have the potential to cause the specimen to drift which affects data quality and acquisition throughput.

Our group has developed an automated system for TEM data acquisition called Leginon [2] that is tightly linked to a relational database [3] which keeps track of all images acquired and the parameters associated with them. Tracking the images using the database provides us with the opportunity of querying the extent to which environmental conditions affect the quality of the acquired data. To achieve this we designed and installed a system for measuring the temperature at a number of points in the microscope room including (i) the TEM column, (ii) the water chiller inlet and outlet pipes, (iii) ambient air, and (iv) the cryoholder. A schematic of the overall architecture of the system is described in Figure 1.

The temperature monitoring system uses National Instrument Fieldpoint [4] modules which are easy-to-use, highly expandable data acquisition and control systems composed of I/O modules and communication interfaces. The FieldPoint allows for measurement, industrial control, and data logging application. The system can communicate to the database over an ethernet interface and thus does not require any additional computing resources in the microscope room. The modules are programmed and controlled using LabVIEW, a graphical development environment [5]. Each module can control up to 8 platinum Resistance Temperature Detectors (RTD), a device which based on the principle of metal resistance increasing with temperature. We are using platinum RTDs from OMEGA [4], which provide very sensitive and repeatable measurements. They have been calibrated to the worldwide accepted DIN 43760 curve (alpha $=0.00385$ ) providing temperatures accurate to $0.3^{\circ} \mathrm{C}$. The location of the temperature sensors is shown schematically in Figure 2. The temperatures are transferred from LabVIEW to the Leginon MySQL database using a PHP script. PHP scripts are also used to produce web pages that can be used to query the temperature (Figure 3 ).

The system is now in regular use and allows us to closely monitor the environment during data acquisition. An automated alarm system sends an email message out to a designated mailing list if the temperature goes outside of specified limits. The database allows us to associate fluctuations in temperature with other measured parameters (for example, specimen drift, images quality). We are currently undertaking a series of systematic experiments designed to determine whether tighter temperature control could improve the quality and the acquisition efficiency of imaging. 


\section{References}

[1] O'Keefe et al. Microscopy Today, 12, (2004): 8-14.

[2] Suloway et al., J. Struct. Biol. (2004), In press.

[3] Fellmann et al., J. Struct .Biology, (2002) 137, 273-282.

[4] National Instrument http://www.ni.com

[5] Labview reference http://www.ni.com

[6] OMEGA http://www.omega.com

[7] This work was conducted at the National Resource for Automated Molecular Microscopy which is supported by the NIH National Center for Research Resource (RR17573).

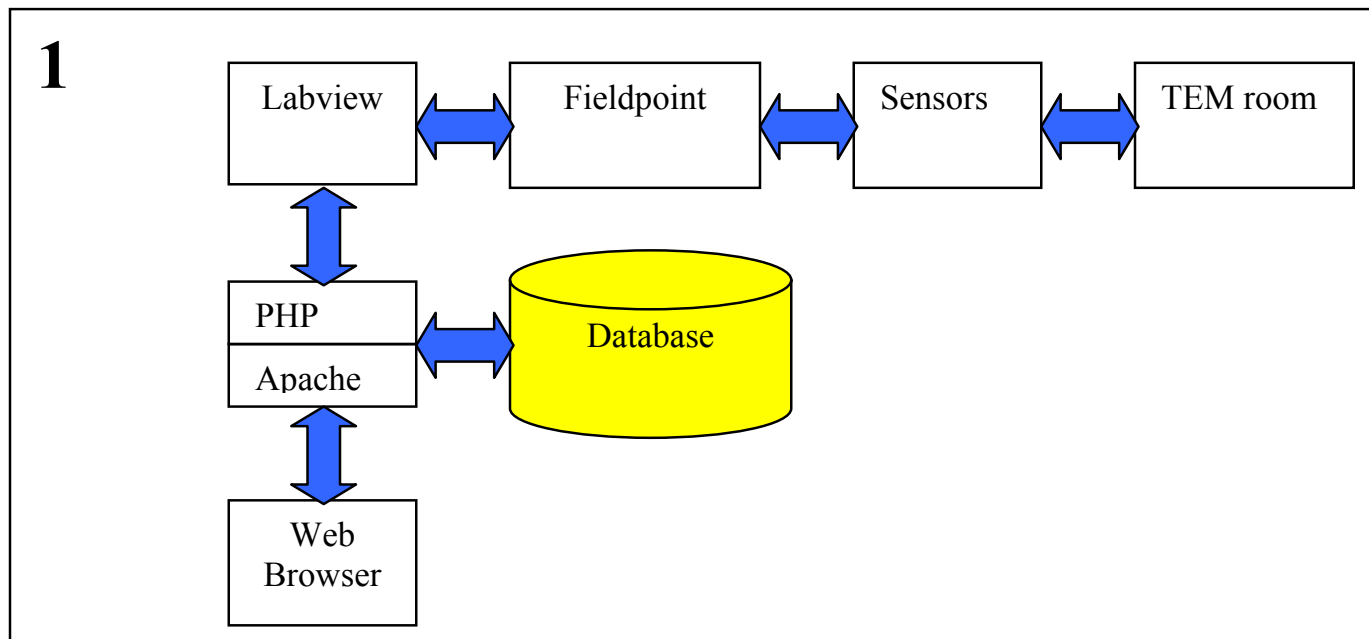

Figure 1: Schematic overview of the environmental monitoring system.

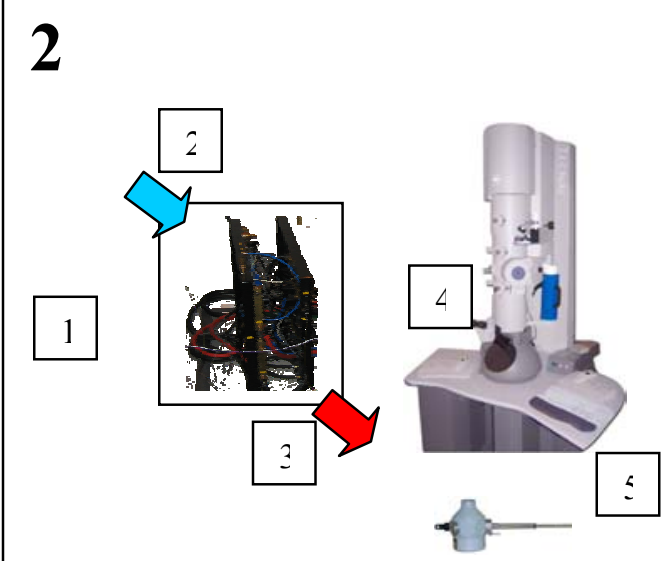

Figure 2: Location of the 5 temperature sensors on our current system.

1: Ambient Air

2: Water cooling IN

3: Water cooling OUT

4: TEM column

5: Cryoholder

\section{3}

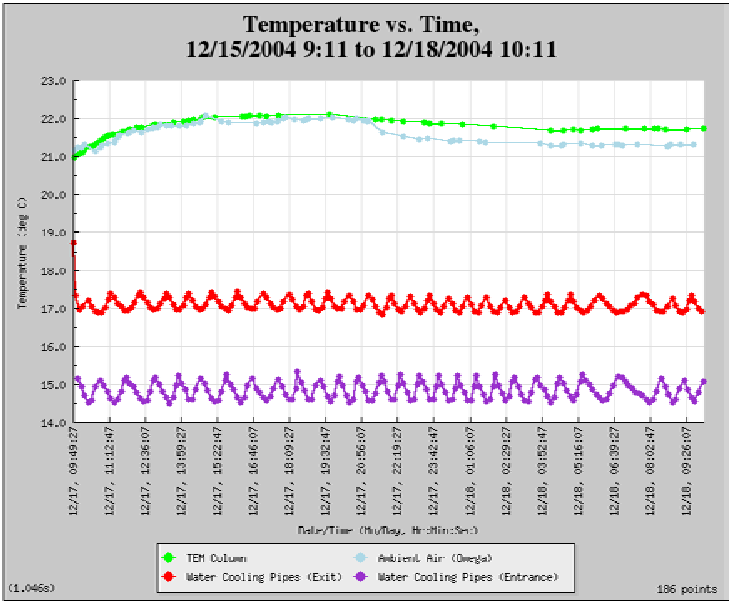

Figure 3: Example of a temperature report generated from web based user interface. 